\title{
Apatinib plus ifosfamide and etoposide for relapsed or refractory osteosarcoma: A retrospective study in two centres
}

\author{
LU XIE ${ }^{1}$, JIE XU $^{1}$, XIN SUN $^{1}$, XIAOWEI LI ${ }^{1}$, KUISHENG LIU $^{1}$, XIN LIANG $^{1}$, ZULI ZHOU $^{2}$,

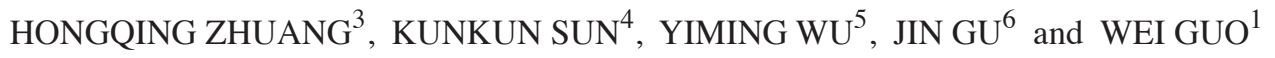 \\ ${ }^{1}$ Musculoskeletal Tumor Center, ${ }^{2}$ Department of Thoracic Surgery, Peking University People's Hospital; \\ ${ }^{3}$ Department of Radiotherapy, Peking University Third Hospital; ${ }^{4}$ Pathology Department, Peking University People's Hospital; \\ ${ }^{5}$ Endocrinology Department, ${ }^{6}$ Department of Surgical Oncology, Peking University Shougang Hospital, \\ Beijing 100044, P.R. China
}

Received February 18, 2021; Accepted April 27, 2021

DOI: $10.3892 / \mathrm{ol} .2021 .12813$

\begin{abstract}
For osteosarcoma that progresses following first-line chemotherapy, prognosis remains poor although anti-angiogenesis tyrosine kinase inhibitors (TKIs) have been verified to prolong progression-free survival. Apatinib has led to positive responses in the treatment of refractory osteosarcoma. However, it demonstrates only short-lived activity, and the disease control rate of musculoskeletal lesions is worse compared with that of pulmonary lesions. This treatment failure has been partly overcome by the addition of ifosfamide and etoposide (IE). The present study retrospectively compared the activity of apatinib + IE in relapsed or refractory osteosarcoma in two sarcoma centres in China. The included patients had received a combination of apatinib $500 \mathrm{mg}$ (orally) daily and the IE regimen ( $\mathrm{n}=33$ ) between June 32017 and July 17 2020. The tumour burden was considerable in these patients: 16/33 (48.5\%) Patients had lung and musculoskeletal lesions, and 31/33 (93.9\%) patients had progressed to two lines of therapies at baseline. With a median follow-up duration of 28.4 [interquartile range (IQR), 16.1-38.3] months, $21 / 33(63.6 \%)$ patients had objective responses, and the
\end{abstract}

Correspondence to: Dr Wei Guo, Musculoskeletal Tumor Center, Peking University People's Hospital, 11 Xizhimen South Street, Xicheng, Beijing 100044, P.R. China

E-mail: bonetumor@163.com

Abbreviations: AEs, adverse events; BSA, body surface area; CI, confidence interval; ECOG, Eastern Cooperative Oncology Group; EFS, event-free survival; ESMO, European Society for Medical Oncology; IE, ifosfamide and etoposide; IFO, ifosfamide; IQR, interquartile range; OS, overall survival; PFS, progression-free survival; PLPS, posterior leukoencephalopathy syndrome; po, orally; Q2w, once every 2 weeks; Q3W, once every 3 weeks; QD, once daily; RECIST 1.1, Response Evaluation Criteria for Solid Tumours; TKIs, tyrosine kinase inhibitors

Key words: apatinib, ifosfamide, etoposide, osteosarcoma, relapsed or refractory, metastasis median event-free survival was 11.4 (IQR, 6.7-18.4) months. The median overall survival time was 19.8 (IQR, 13.1-30.6) months. At the last follow-up, 16/33 patients had tumour downstaging, and all lesions had been completely resected. For osteosarcoma with multiple sites of metastasis, apatinib + IE demonstrated clinically meaningful antitumor activity and delayed disease progression in patients with recurrent or refractory osteosarcoma after failure of chemotherapy. This combination with manageable toxicity deserves further investigation in prospective trials.

\section{Introduction}

Osteosarcoma is a highly aggressive sarcoma that originates from mesenchymal stem cells with osteoblastic lineage commitment, for which the treatment regimen has remained essentially unchanged for $>40$ years $(1,2)$. The 5 -year overall survival (OS) of advanced osteosarcoma refractory to multiagent chemotherapy has remained $<20 \%$ over decades according to recent European and American prospective trial results $(1,2)$. Although anti-angiogenesis tyrosine kinase inhibitors (TKIs) have been shown to prolong 6-month progression-free survival rate (PFSR) in heavily treated patients in multiple trials, patients have a median overall survival of $<12$ months, and a standard management strategy has yet to be established (3-7). Apatinib, a multikinase inhibitor used in gastric, non-small cell lung and oesophageal cancer, has been shown to prevent the proliferation and migration/invasion of cell lines and subsequent growth and metastasis in various osteosarcoma preclinical studies (8-10). These findings led to two phase 2 trials to explore the activity of apatinib-based treatment in patients with relapsed, unresectable high-grade osteosarcoma after standard treatment (11). In this setting, apatinib showed signs of antitumor activity in terms of the proportion of patients who achieved a response (43\%). However, these encouraging results were short lived, with only $36.8 \%$ of patients were progression free at 6 months. The combination regimen of camrelizumab, a humanized IgG4-k PD-1 monoclonal antibody, and apatinib did not significantly prolong PFS compared with apatinib alone (11-13). From these prospective trials, it was observed that different target lesions at different locations responded distinctly to apatinib, with a 
varied duration of response. Moreover, musculoskeletal lesions were found to quickly develop secondary resistance to apatinib, with a median PFS time of only 2.1 [95\% confidence interval (CI) 1.8-5.7] months compared with pulmonary lesions $(12,13)$. Furthermore, nearly one-third of the cases of progression were due to musculoskeletal lesions, while another one-third were due to the appearance of new lesions outside the lung (11-13). Thus, overcoming disease progression outside the lung during TKI-based treatment has become an important clinical challenge.

Ifosfamide (IFO) is one of the most active systemic agents for the treatment of patients with sarcoma $(14,15)$. In addition to its anticancer activity, ifosfamide is a radiosensitizing agent that can be given concurrently with radiotherapy (15). Etoposide (VP16) was one of the first identified topoisomerase II inhibitors and has been studied for decades in combination with IFO for treating osteosarcoma (16). However, the findings of the European and American Osteosarcoma Study did not support the addition of ifosfamide and etoposide (IE) to postoperative chemotherapy in poorly responding osteosarcoma because of increased toxicity and no improvement in event-free survival (17). Despite this finding, the status of IE in osteosarcoma is ambiguous between first- and second-line chemotherapy (18-22), and several sarcoma centres have still merged this agent into first-line chemotherapy regimens for various reasons. Gaspar et al (23), at the 2019 Congress of the European Society for Medical Oncology (ESMO), proposed a combination of lenvatinib and IE as a second-line therapy for recurrent osteosarcoma; this therapy was found to prolong the median PFS from 3 (95\% CI, 1.8-5.5) months with single lenvatinib to 11.1 (95\% CI, 4.5-12.6) months. As a result, it was speculated that the addition of chemotherapy overcame the disadvantage of the poor disease control of musculoskeletal lesions with TKIs. Therefore, the present study sought to evaluate the combination strategy of apatinib + IE in patients with recurrent or refractory osteosarcoma from retrospective data, especially those with metastatic lesions both in and outside the lung. The current study was designed to review our experience and investigate the activity of this combination therapy in these patients in two sarcoma centres in China and to further characterize the toxicity profile of this combination in Asian patients.

\section{Patients and methods}

Patients. From June 32017 to July 17 2020, patients who received apatinib + IE were included if they met the following criteria: i) Histologically confirmed high-grade osteosarcoma according to Enneking Grading System (24); ii) initial treatment in the Orthopaedic/Oncology Departments of Peking University People's Hospital or Peking University Shougang Hospital (both Beijing, China); iii) progression $<6$ months after first-line chemotherapy (25) with a combination of high-dose methotrexate, doxorubicin, cisplatin and ifosfamide (Fig. 1) (MAPI), including high-dose methotrexate $\left(12 \mathrm{~g} / \mathrm{m}^{2}\right)$, doxorubicin $\left(75 \mathrm{mg} / \mathrm{m}^{2}\right)$, cisplatin (100-120 mg/m²) with or without ifosfamide $\left(12 \mathrm{~g} / \mathrm{m}^{2}\right)$. We usually defined these four agents as first-line chemotherapy while choose IE as the second-line chemotherapy, which included ifosfamide $1.8 \mathrm{~g} / \mathrm{m}^{2} / \mathrm{d} \mathrm{d}_{1-5}$ and etoposide $100 \mathrm{mg} / \mathrm{m}^{2} / \mathrm{d}$ $\mathrm{d}_{1-5}$ Q3W (IE); iv) measurable lesions according to the Response Evaluation Criteria for Solid Tumours (RECIST 1.1) (26); v) Eastern Cooperative Oncology Group (ECOG) performance status $\leq 2$ (27); and vi) acceptable haematological, hepatic and renal function. The following patients were excluded: i) Those who did not have intact clinical evaluation radiographic materials or complete follow-up information after chemotherapy; ii) those who did not receive more than two courses of the regimens due to reasons other than tumour progression or toxicity; and iii) those who had severe or uncontrolled medical disorders that could jeopardize the outcomes of the study. Notably, patients who had previously shown progression upon single apatinib or IE and then later adopted combination therapy were included to analyse whether this combination had synergistic effects. All patients who had been assessed by the joint sarcoma board of the aforementioned hospitals, which contains medical, paediatric, surgical and radiology oncologists in both hospitals, for eligibility for metastasectomy were also included. It was possible for the included patients to undergo surgery with curative intent only if they had been stable for at least 4 months.

This trial was retrospectively registered in The Medical Ethics Committees of Peking University People's Hospital and Peking University Shougang Hospital on December 282020 (registration no. 2020PHB388-02). The trial was registered at ClinicalTrials. gov under identifier no. NCT04690231. Both hospitals obtained approval from The Medical Ethics Committee of Peking University People's Hospital and The Medical Ethics Committee of Peking University Shougang Hospital (Beijing, China) to review the patients' medical records and radiographic materials. The outcome data were then retrospectively combined. Written informed consent from patients was not required.

During systemic treatment, patients underwent chest computed tomography (CT) or $\mathrm{CT} /$ magnetic resonance imaging of the musculoskeletal tumour sites every 2 months. Following the completion of chemotherapy, patients were followed-up every 2 months for the first 2 years and then every 3 months for the next 3 years with median follow-up time of 19.8 (IQR, 9.4,51.8) months. Radionuclide bone scans or positron emission tomography/CT was used to assess metastatic disease every 6 months during treatment and for the first 5 years after the completion of systemic treatment. All the adverse events (AE) which were deemed to be associated with study drugs were recorded according to according to Common Terminology Criteria for Adverse Events (CTCAE) version 5.0 (28). For this retrospective study, we recorded severe AEs related to various dose combinations in Table II, of which we sometimes hardly verdicted whether the AEs were related to TKIs or chemotherapy.

Treatment protocols. Initially, IE chemotherapy was routinely administered as follows: Ifosfamide $1.8 \mathrm{~g} / \mathrm{m}^{2} / \mathrm{d} \mathrm{d}_{1-5}$ and etoposide $100 \mathrm{mg} / \mathrm{m}^{2} / \mathrm{d} \mathrm{d}_{1-5}$ once every 2 weeks (Q2w), with sufficient hydration and mesna protection. Given the variability in the disease status and haematopoietic function of the patients, for the combination therapy, patients were retrospectively divided into five groups for IE dosing (more than half the duration of the whole treatment course) and three groups for apatinib dosing as follows: $250 \mathrm{mg}$ once daily (QD) orally (po) [body surface area (BSA) $\geq 1.0$ ]; $500 \mathrm{mg}$ QD po (BSA $\geq 1.0$ ) or $250 \mathrm{mg}$ QD po (BSA $<1.0$ ); and $375 \mathrm{mg}$ QD po (BSA $\geq 1.0$ ). For the combination therapy we had tried the following combinations: i) Ifosfamide $2.4 \mathrm{~g} / \mathrm{m}^{2} / \mathrm{d} \mathrm{d}_{1-5} \mathrm{Q} 3 \mathrm{~W}$, ii) ifosfamide $2.0 \mathrm{~g} / \mathrm{m}^{2} / \mathrm{d} \mathrm{d}_{1-5} \mathrm{Q} 3 \mathrm{~W}$ and iii) ifosfamide $1.8 \mathrm{~g} / \mathrm{m}^{2} / \mathrm{d} \mathrm{d}_{1-5}$, etoposide $100 \mathrm{mg} / \mathrm{m}^{2} / \mathrm{d} \mathrm{d}_{1-5} \mathrm{Q} 3 \mathrm{~W}$. Next, the 
Protocol for osteosarcoma-02 in PKUPH

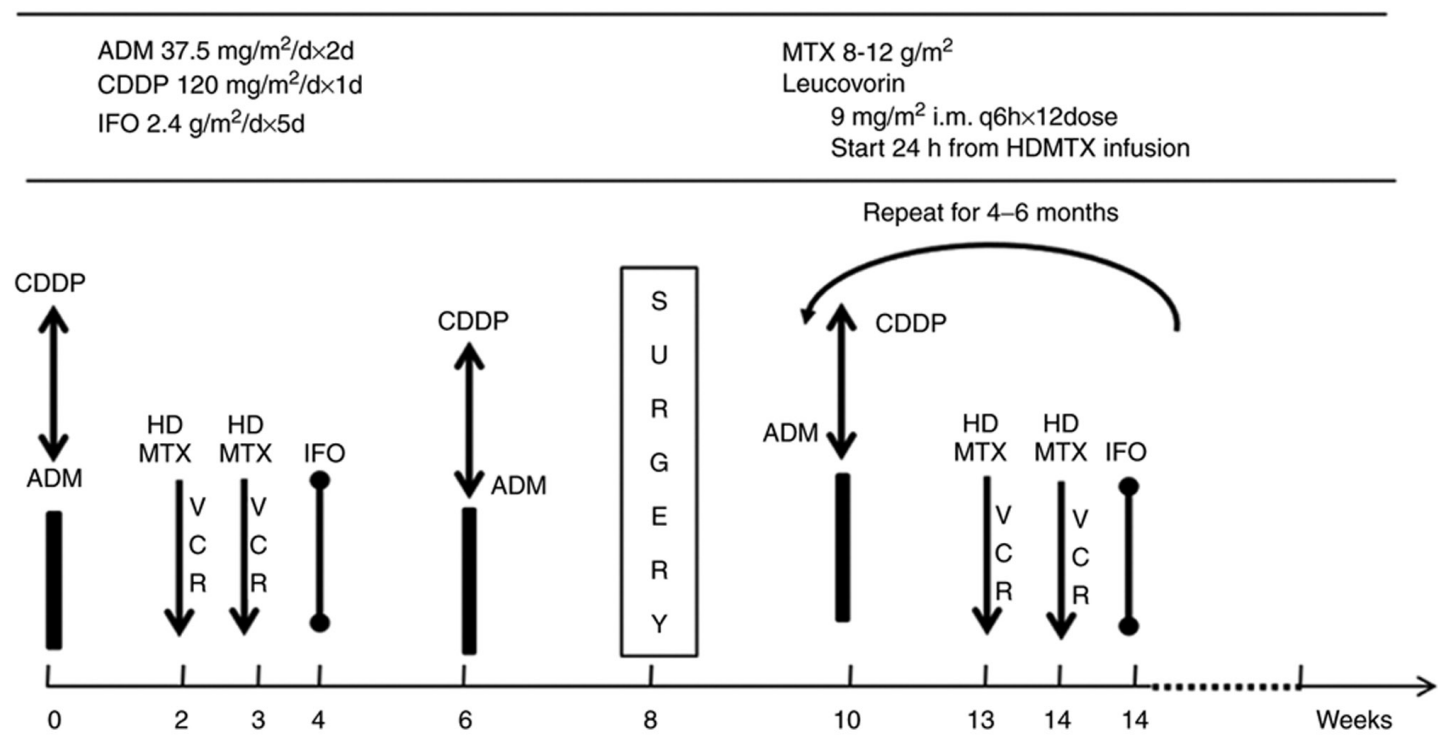

Figure 1. Pictured is the updated first-line osteosarcoma regimen used at PKUPH. ADM, doxorubicin; CDDP, cisplatin; DOX, doxorubicin; HD MTX high-dose methotrexate; IFO, ifosfamide; VCR, vincristine; PKUPH, Peking University People's Hospital.

following combinations were attempted: iv) Ifosfamide $1.8 \mathrm{~g} / \mathrm{m}^{2} / \mathrm{d} \mathrm{d}_{1-3}$; etoposide $100 \mathrm{mg} / \mathrm{m}^{2} / \mathrm{d} \mathrm{d}_{1-3} \mathrm{Q} 2 \mathrm{w}$ and in some patients v) ifosfamide $1.8 \mathrm{~g} / \mathrm{m}^{2} / \mathrm{d} \mathrm{d}_{1-3} \mathrm{Q} 2 \mathrm{w}$. Severe haematological toxicity was classified as the following: i) Haematological toxicity: Absolute neutrophil count $<500 / \mathrm{mm}^{3}$ for $\geq 3$ days and platelets $<25,000 / \mathrm{mm}^{3}$ for $\geq 3$ days despite platelet transfusion or grade $\geq 3$ thrombocytopenic bleeding and ii) non-haematological grade $\geq 3$ toxicities. If these were observed in patients then VP16 was removed from the treatment regime following the dose reduction protocols of COG protocol-AOST0331 as well as EURAMOUS-1 (17).

Statistical analysis. All analyses were performed with SPSS 19.0 software (IBM Corp.). The data were presented as n (\%) or median, interquartile range. Given that some patients underwent lesion resection, event-free survival (EFS) was calculated from the date at which apatinib + IE (not from first-line chemotherapy) was started until disease progression or death, whichever came first in median value as well as interquartile range. PFS was calculated from the start of target treatment until disease progression or death, whichever came first. For patients who underwent lesion resection, the events were calculated as censored for PFS and recorded the time at the local therapeutic time point. Overall survival (OS) was calculated from the date of treatment initiation to death from any cause. Descriptive statistics were used to display demographic data. Kaplan-Meier plotter was used to determine OS, EFS and PFS (29). Cox proportional hazards analysis was subsequently performed on variables to identify factors associated with survival and local recurrence. $\mathrm{P}<0.05$ was considered to indicate a statistically significant difference.

\section{Results}

Patient characteristics. In total, 33 patients who received 818 courses of apatinib + IE were identified, among whom the initial diagnosis was established between August 2014 and
January 2020. The characteristics of the included patients are summarized in Table I. Patients' age were grouped according to Collins et al (30) in Table I because in EURAMOUS-1 trial this factor significantly influenced the outcomes (17). In total, 13/33 (39.4\%) patients had ECOG performance status scores of 1 or 2, and $17 / 33(51.5 \%)$ had lesions located in the lungs, as well as musculoskeletal sites or viscera. In addition, $18 / 33(54.6 \%)$ had progressed on two or more lines of systemic therapy at baseline. Notably, 12/33 (36.4\%) patients progressed on IE chemotherapy, among whom 2/33 (6.1\%) had progressed on apatinib alone (Table I). Usually, these cases of progression manifested as indolent progression patterns, such as PD over a time window of 4-6 months or even longer based on the RECIST 1.1 or simply oligoprogression of 1-2 lesions (data not shown). Of the patients who had ever progressed on IE chemotherapy, all still had the potential to subsequently benefit from the combination.

Dosing. For the combination therapy we had tried the following combinations i), ii) and iii). However, only two patients could tolerate dosing i) and one patient could tolerate dosing ii). The majority of patients had myelosuppression that was so severe that the doses were reduced shortly after initiation (after 1-2 cycles). Then we tried dose iv) and v), of which the patients' tolerance was improved (14/33 and 13/33 of the population, respectively). It was noticed that $4 / 33$ (12.1\%) of patients with unresectable pulmonary and musculoskeletal lesions had been given doses iv and iii of IE (one and three patients, respectively) for $>12$ months without disease progression or severe toxicity (12.6, 13.9, 18.4 and 19.2 months) (data not shown). However, after 8 to 9 months of treatment, the intervals of their chemotherapy cycles were sometimes prolonged to 3-4 weeks sometimes due to myelosuppression and sometimes just patients' preferences. Two patients had been so heavily treated that dosing iv was attempted, but treatment was interrupted after just 3 cycles due of myelosuppression. Then, dose $v$ was chosen for 2 and 6 months (for each patient). Due to the 
Table I. Demographics of 33 patients with osteosarcoma treated with apatinib+IE in the study.

\begin{tabular}{|c|c|}
\hline Variable & Value \\
\hline Age, median years (minimum, maximum) & $16.0(3.0,48.0)$ \\
\hline \multicolumn{2}{|l|}{ Age group ${ }^{\mathrm{a}}, \mathrm{n}(\%)$} \\
\hline Child & $8(24.2)$ \\
\hline Adolescent & $9(27.3)$ \\
\hline Adult & $16(48.5)$ \\
\hline \multicolumn{2}{|l|}{ Sex, n $(\%)$} \\
\hline Male & $14(42.4)$ \\
\hline Female & $19(57.6)$ \\
\hline \multicolumn{2}{|l|}{ ECOG performance status before treatment, n (\%) } \\
\hline 0 & $20(60.6)$ \\
\hline 1 & $11(33.3)$ \\
\hline 2 & $2(6.1)$ \\
\hline \multicolumn{2}{|l|}{ Sites of target and non-target lesions before treatment, n (\%) } \\
\hline Lung only & $13(39.4)$ \\
\hline Musculoskeletal sites only & $3(9.1)$ \\
\hline Lung + musculoskeletal sites & $16(48.5)$ \\
\hline Lung + bone + other sites ${ }^{\mathrm{b}}, \mathrm{n}(\%)$ & $1 \quad(3.0)$ \\
\hline \multicolumn{2}{|l|}{ Lines of previous chemotherapy including MAP/I ${ }^{\mathrm{c}}, \mathrm{n}(\%)$} \\
\hline 1 & $15(45.4)$ \\
\hline 2 & $16(48.5)$ \\
\hline 3 & $2(6.1)$ \\
\hline \multicolumn{2}{|l|}{ High-grade osteosarcoma subtypes, n (\%) } \\
\hline Canonical (osteoblastic, chondroblastic, fibroblastic) & $32(97.0)$ \\
\hline Small cell & $0 \quad(0.0)$ \\
\hline Telangiectatic & $1 \quad(3.0)$ \\
\hline \multicolumn{2}{|l|}{ Resistance to MAP/I chemotherapy ${ }^{\mathrm{d}}, \mathrm{n}(\%)$} \\
\hline Yes & $33(100.0)$ \\
\hline No & $0 \quad(0.0)$ \\
\hline \multicolumn{2}{|l|}{ Resistance to IE chemotherapy ${ }^{\mathrm{e}}, \mathrm{n}(\%)$} \\
\hline Yes & $12(36.4)$ \\
\hline No & $21(63.6)$ \\
\hline \multicolumn{2}{|l|}{ Resistance to apatinib ${ }^{\mathrm{f}}, \mathrm{n}(\%)$} \\
\hline Yes & $2 \quad(6.1)$ \\
\hline No & 31 (93.9) \\
\hline
\end{tabular}

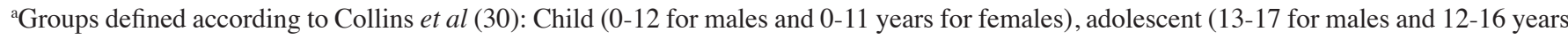

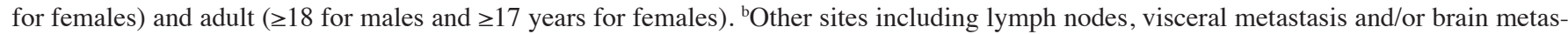
tasis. ${ }^{\mathrm{M}} \mathrm{MP} / \mathrm{I}$, including high-dose methotrexate $\left(12 \mathrm{~g} / \mathrm{m}^{2}\right)$, doxorubicin $\left(75 \mathrm{mg} / \mathrm{m}^{2}\right)$, cisplatin $\left(100-120 \mathrm{mg} / \mathrm{m}^{2}\right)$ with or without ifosfamide $\left(12 \mathrm{~g} / \mathrm{m}^{2}\right)$. ${ }^{\mathrm{d}}$ Resistance to MAP/I chemotherapy based on RECIST 1.1, patients who exhibited disease progression upon MAP/I chemotherapy.

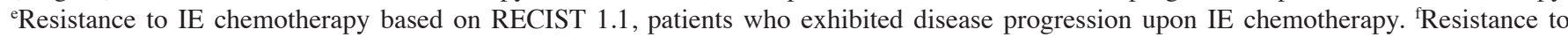
apatinib based on RECIST 1.1, patients who exhibited disease progression upon single apatinib treatment. ECOG, Eastern Cooperative Oncology Group; IE, ifosfamide and etoposide; MAP/I, high-dose methotrexate, doxorubicin, cisplatin with or without ifosfamide; RECIST, Response Evaluation Criteria for Solid Tumours.

complexity of the data and given that this was a retrospective investigation, we then summarized the dosing for patients who received for more than half of the treatment course as their recorded dosing into Table II. It was noticed that for the heavily treated patients with a high tumour burden in multiple metastatic sites, combinations with a low dose and long dosing times resulted in improved clinical benefit. Thus, we aimed not to use aggressive treatment to induce an objective response and then interrupt treatment because of toxicity but rather to use the combination of a similar metronomic chemotherapy and TKIs to prolong overall disease control.

Assessment of efficacy. With a median follow-up of 19.8 [interquartile range (IQR), 10.1-29.4] months, 20/79 (25.3\%) 


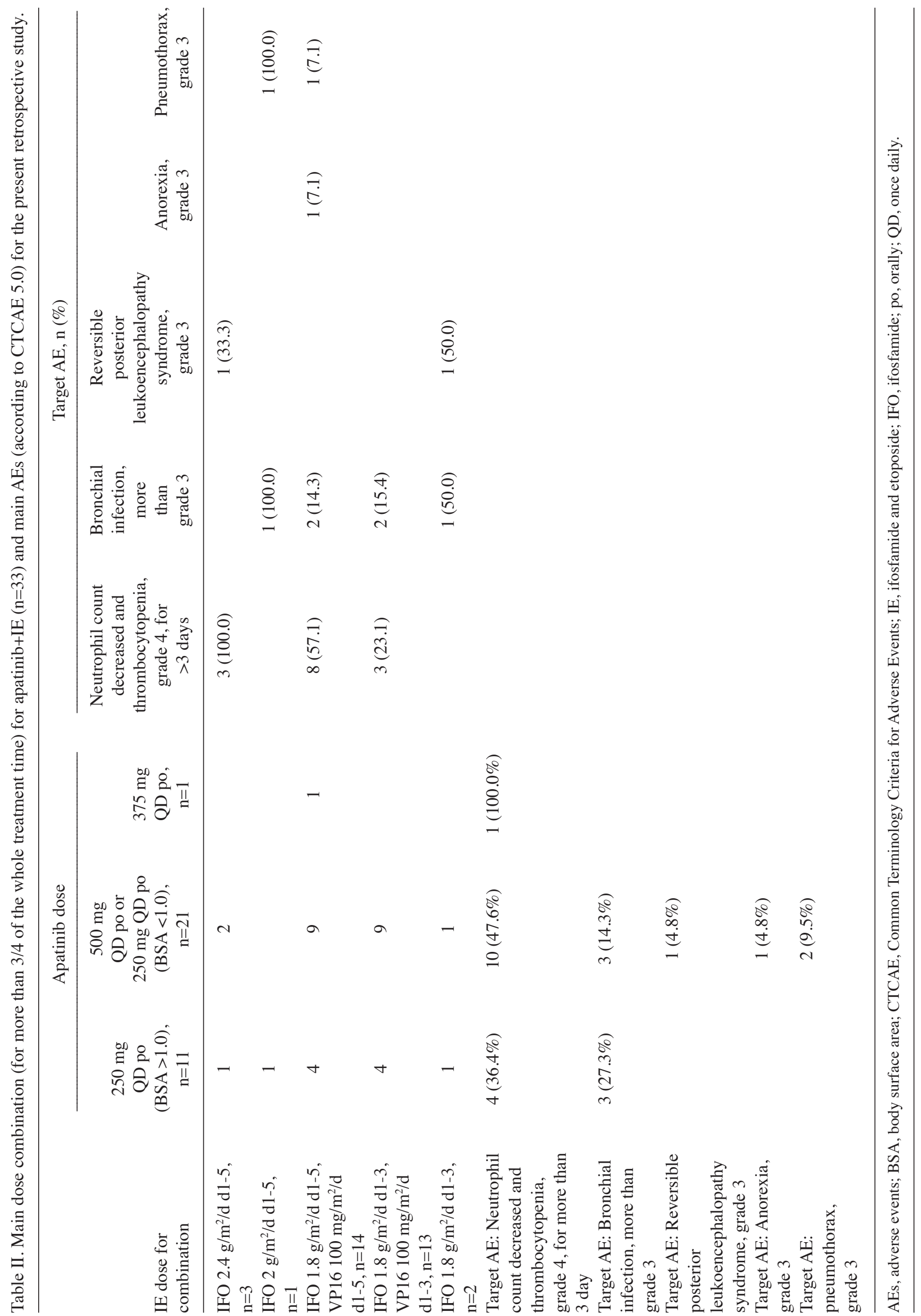




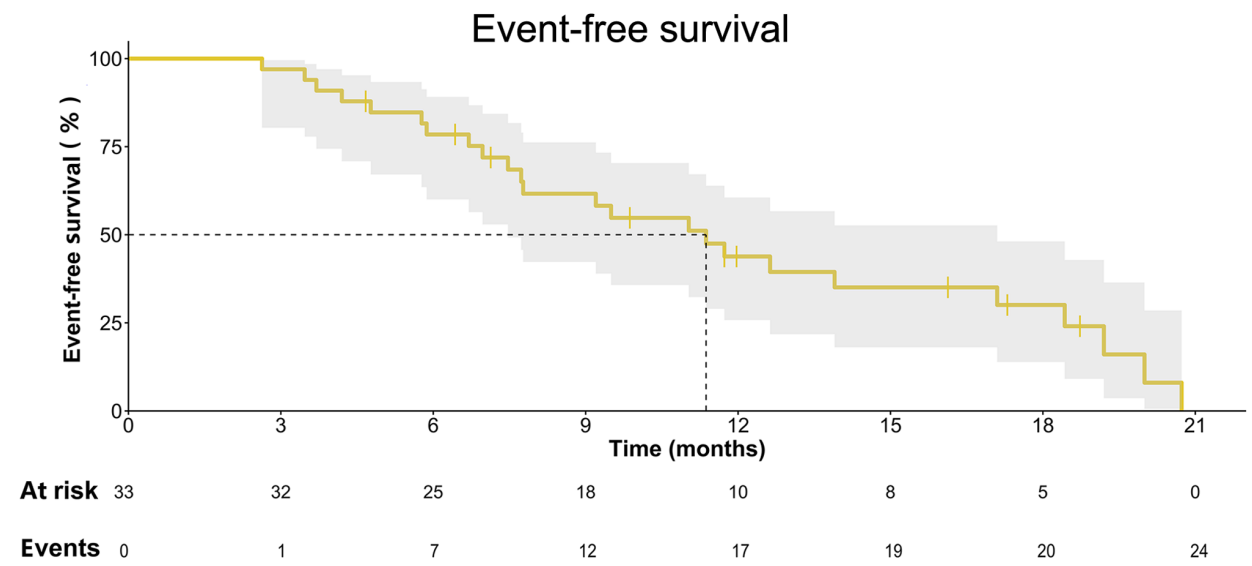

Figure 2. Kaplan-Meier plots for event-free survival in 31 patients with advanced osteosarcoma receiving apatinib + ifosfamide and etoposide chemotherapy. Crosses indicate censored data.

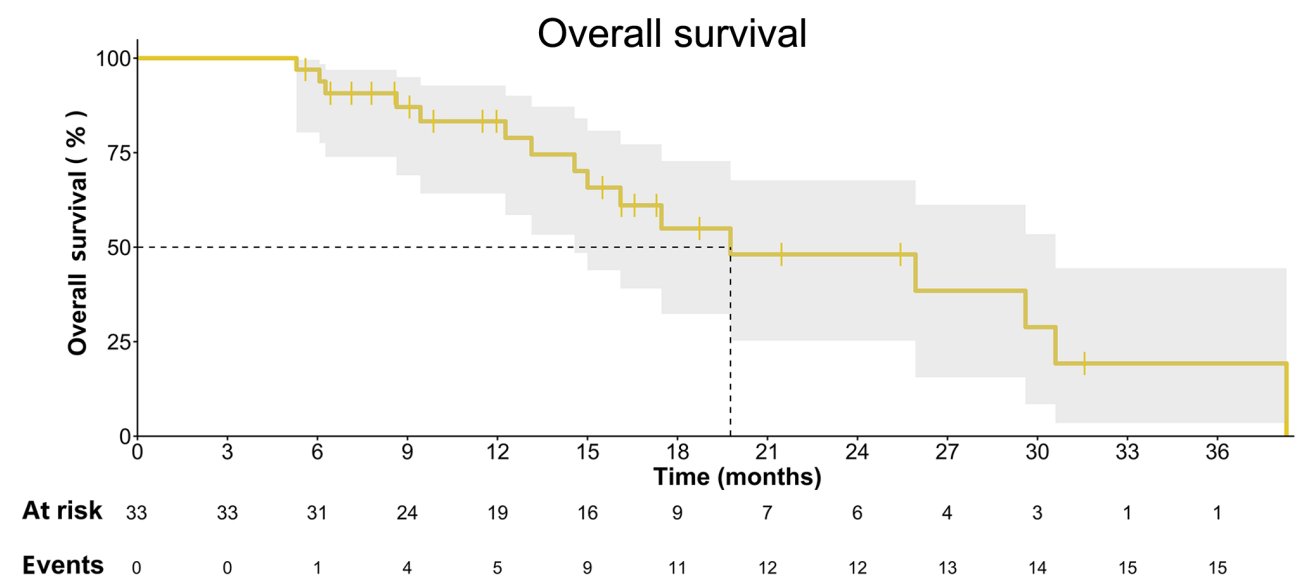

Figure 3. Kaplan-Meier plot for overall survival of 31 patients with advanced osteosarcoma receiving apatinib + ifosfamide and etoposide chemotherapy. Crosses indicate censored data.

patients showed no evidence of disease, $27 / 79$ (34.2\%) patients were alive with disease, and 32/79 (40.5\%) patients died of disease (Table II, Figs. 2 and 3). Thus, the OS data were estimated using Kaplan-Meier analysis. As of the most recent follow-up, 21/33 (63.6\%) patients had partial responses, while 12/33 (36.4\%) had stable disease without any initial disease progression at the first 2-month evaluation (Table III) according to the RECIST 1.1.

The 4- and 6-month EFS rates were $90.9 \%$ (95\% CI, $74.4-97.0 \%$ ) and $78.5 \%$ (95\% CI, 60.0-89.1\%), respectively, with a median EFS of 11.4 (IQR, 7.5-17.1) months (Fig. 2). The median PFS time was 12.6 (95\% CI, 6.4-18.9) months, with 22/33 (66.7\%) events censored (Fig. S1). At the last follow-up, $16 / 33$ patients had tumour downstaging, and all lesions had been completely resected (Table III). Thus, EFS was more representative of the real-world conditions of our patients compared with OS. The median OS time of these patients was 19.8 (IQR, 10.1-29.4) months (Fig. 4). We then compared the data of our previous clinical trials' data for single apatinib and apatinib in combination with camrelizumab, as well as our retrospective data of single IE chemotherapy delivered from 2016 to 2019 into Table IV. However, even for the most heavily treated patient group with the highest tumour burden, the combination of apatinib and IE chemotherapy led to a more objective tumour response (Fig. 4) and a higher disease control rate (Table IV) than compared with IE chemotherapy, single apatinib or apatinib + camrelizumab.

Toxicity and safety. The severe adverse events (AEs) related to various dose combinations are summarized in Table II according to CTCAE v.5.0, of which the dosing used for more than half of the treatment course was recorded. A total of 25 severe AEs were recorded. Despite the fact that the daily dose of apatinib was lower than that used in the aforementioned phase II trial (11), the AEs were both higher grades and different compared with those in the treatment combination with IE. The majority of grade 3 and 4 toxicities were neutropenia, thrombocytopenia, bronchial infection, pneumothorax, anorexia, and posterior leukoencephalopathy syndrome (PLPS). It is worth noting that the records for AEs might not have been complete given the retrospective nature of the study. However, physicians are trained to record the most concerning AEs to empirically deliver regimens safely. From the data shown in Table II, it is speculated that the dose combination with apatinib $500 \mathrm{mg}$ QD po for BSA $\geq 1.0$ and IFO $1.8 \mathrm{~g} / \mathrm{m}^{2} / \mathrm{d} \mathrm{d}_{1-5} \mathrm{VP} 16100 \mathrm{mg} / \mathrm{m}^{2} / \mathrm{d} \mathrm{d}_{1-3} \mathrm{Q} 2 \mathrm{w}$ could represent a compromise, with acceptable toxicities in these patients. 


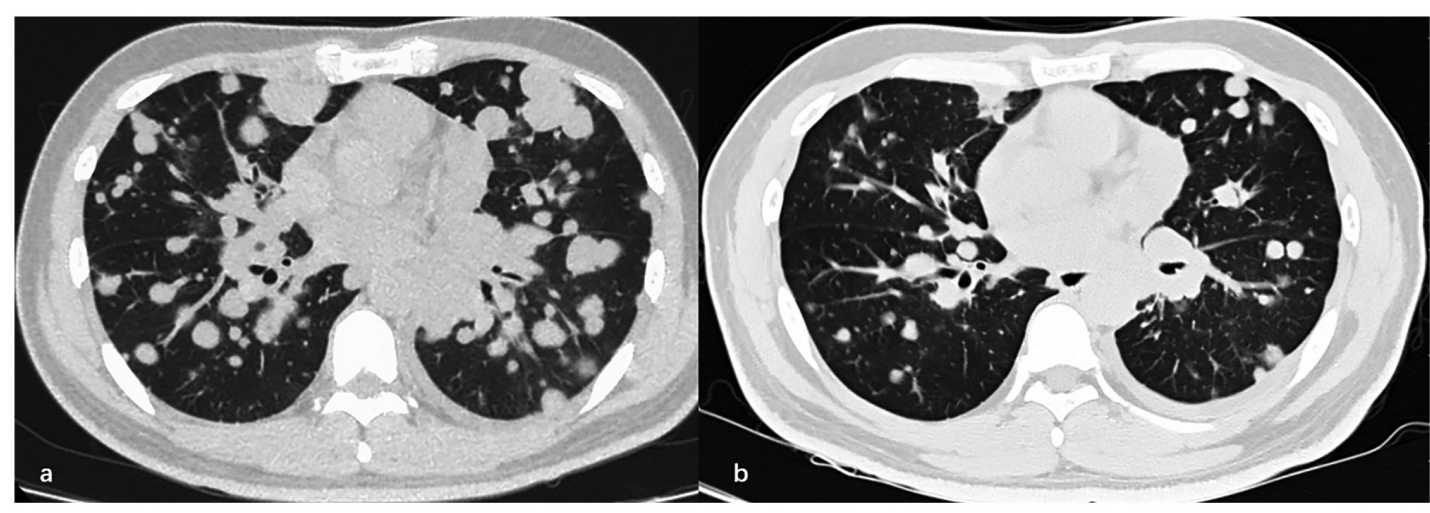

Figure 4. Chest computed tomography of a 30-year male with multiple pulmonary metastases and a high tumour burden (a) before treatment and (b) after two cycles of apatinib plus ifosfamide and etoposide (IE) chemotherapy.

Table III. Efficacy of combination treatment of apatinib+IE in patients 33 with advanced osteosarcoma.

\begin{tabular}{lc}
\hline Efficacy variable & Value \\
\hline Complete response, $\mathrm{n}(\%)$ & $0(0.0)$ \\
Partial response, $\mathrm{n}(\%)$ & $21(63.6)$ \\
Stable disease, $\mathrm{n}(\%)$ & $12(36.4)$ \\
Progressive disease, $\mathrm{n}(\%)$ & $0(0.0)$ \\
CBR of ITT, 6 months (95\% CI) & $81.8 \%(68.7 \%, 95.0 \%)$ \\
ITT event-free survival & \\
KM, median (95\% CI) & $11.4(7.5,17.1)$ \\
4 months, n\% (95\% CI) & $90.9(74.4,97.0)$ \\
6 months, n\% (95\% CI) & $78.5(60.0,89.1)$ \\
ITT overall survival & \\
KM, median, IQR & $19.8(10.1,29.4)$ \\
Patient status at last follow-up, n (\%) & \\
NED & $4(12.1)$ \\
AWD & $13(39.4)$ \\
DOD & $16(48.5)$ \\
Complete surgical remission, $\mathrm{n}(\%)$ & $16(48.5)$ \\
\hline
\end{tabular}

AWD, alive with disease; CBR, clinical benefit rate (6 months); CI, confidence interval; DCR, disease control rate; DOD, died of disease; IE, ifosfamide and etoposide; IQR, interquartile range; ITT, intention-to-treat population; KM, Kaplan Meier; MAP/I, high-dose methotrexate, doxorubicin, cisplatin with or without ifosfamide; NED, no evidence of disease.

\section{Discussion}

The prognosis of patients with recurrent or refractory osteosarcoma progression upon first-line chemotherapy has been predominantly poor for the past 30 years with 5 -year post-relapse survival in $<20 \%$ globally (31). Despite evidence of genomic instability characterized by widespread and recurrent somatic copy-number alterations and structural rearrangements, osteosarcoma has few recurrent point mutations in protein-coding genes (32). Moreover, trials of targeted agents have generally been disappointing with median PFS of only 4 to 6 months and secondary drug resistance seemed to be inevitable $(2,4)$. We previously showed the promising anti-angiogenic therapeutic effects of TKIs, including apatinib (11), cabozantinib (33), lenvatinib (22), regorafenib (34) and sorafenib (35), of which the treatment targets are thought to include VEGFRs and proto-oncogene tyrosine-protein kinase receptor RET (a transmembrane receptor protein-tyrosine kinase that is required for the development of the nervous system and several other tissues) (5), in osteosarcoma. However, these TKIs have short-lived activity in the treatment of musculoskeletal lesions or other metastases outside the lungs $(11,13)$. It is suspected that the plasma concentrations of these TKIs might be limited due to their pharmacokinetic characteristics (7). Despite this speculation, very little is known about the biology underlying this poor control of bone lesions. However, it is known that in the early stages of metastatic lung colonization, disseminated cancer cells experience a variety of cellular stresses, including redox/endoplasmic reticulum stress, which threaten their survival in the distant bone microenvironment (36). Thus, overcoming cancer progression outside the lung during TKI-based treatment is important. Moreover, for heavily treated patients with inoperable lesions, AEs need to be managed to a tolerable level given that therapy is potentially lifelong; this leads us to question which types of dose combinations would be most beneficial to this patient group.

The rationale for the combination of apatinib and IE in the present study was built on the theory that the addition of chemotherapy could overcome the weakness of the cytostatic properties of these molecular targeted agents. The results on lenvatinib and IE reported at the 2019 ESMO (23) showed promise for the future use of this combination, of which the median PFS was prolonged to 11.1 months. The current retrospective study also provided evidence for exploration of the activity of apatinib + IE in the treatment of metastatic osteosarcoma with lesions outside the lungs. Indeed, several prospective trials with apatinib-based treatment have been completed in the last decade $(11,13)$. Thus, to compare the efficacy of these regimens, we combined the data of these non-randomized, historical non-controlled cohorts, as well as our retrospective data of single IE chemotherapy delivered from 2016 to 2019 into Table IV. As a result, we were able to observe that different tumour burdens and diverse tumour locations have the potential to significantly influence the treatment outcomes. For advanced osteosarcoma, in our opinion, the 
Table IV. Comparison of the patients' demographics and efficacy of different apatinib-based therapeutic strategies for advanced osteosarcoma.

\begin{tabular}{|c|c|c|c|c|}
\hline Items & $\begin{array}{l}\text { Apatinib+IE, } \\
\mathrm{n}=33\end{array}$ & $\begin{array}{l}\text { Apatinib (11), } \\
\mathrm{n}=37\end{array}$ & $\begin{array}{c}\text { Apatinib+Camrelizumab } \\
\qquad(12,13), \mathrm{N}=41\end{array}$ & $\mathrm{IE}, \mathrm{n}=46$ \\
\hline Study type (11-13) & $\begin{array}{l}\text { Retrospective } \\
\text { study }\end{array}$ & $\begin{array}{l}\text { Prospective } \\
\text { trial }\end{array}$ & $\begin{array}{l}\text { Prospective } \\
\text { trial }\end{array}$ & $\begin{array}{l}\text { Retrospective } \\
\text { study }\end{array}$ \\
\hline Trial registration number & NCT04690231 & NCT02711007 & NCT03359018 & NCT04690231 \\
\hline $\begin{array}{l}\text { Patient age, average years } \pm \\
\text { standard deviation }(95 \% \mathrm{CI})\end{array}$ & $\begin{array}{c}19.1 \pm 8.5 \\
(16.0,22.2)\end{array}$ & $\begin{array}{c}21.7 \pm 11.5 \\
(17.9,25.6)\end{array}$ & $\begin{array}{c}19.7 \pm 9.0 \\
(17.1,22.4)\end{array}$ & $\begin{array}{c}17.7 \pm 9.3 \\
(14.9,20.5)\end{array}$ \\
\hline \multicolumn{5}{|l|}{ Target lesions before treatment, n (\%) } \\
\hline Pulmonary lesions & $13(39.39)$ & $27(72.97)$ & $18(41.86)$ & $39(84.78)$ \\
\hline Musculoskeletal lesions & $3(9.09)$ & $4(10.81)$ & $3(6.98)$ & $1(2.17)$ \\
\hline Lung+ musculoskeletal lesions & $16(48.48)$ & $6(16.22)$ & $22(51.16)$ & $6(13.04)$ \\
\hline Lung+bone+other lesions ${ }^{\mathrm{a}}$ & $1(3.03)$ & $0(0.00)$ & & $0(0.00)$ \\
\hline \multicolumn{5}{|l|}{ Lines of systemic therapy, n (\%) } \\
\hline 1st line $\mathrm{MAPI}^{\mathrm{b}}$ & $15(45.45)$ & $31(83.78)$ & $37(86.05)$ & $40(86.96)$ \\
\hline 2nd line IE/GT & $16(48.48)$ & $5(13.52)$ & $6(13.95)$ & $5(10.87)$ \\
\hline 3rd line & $2(6.06)$ & $1(2.70)$ & & $1(2.17)$ \\
\hline \multicolumn{5}{|l|}{ ECOG status before treatment, $\mathrm{n}(\%)$} \\
\hline 0 & $20(60.60)$ & $27(72.97)$ & $34(79.07)$ & $44(95.65)$ \\
\hline 1 & $11(33.33)$ & $10(27.03)$ & $9(20.93)$ & $2(4.35)$ \\
\hline 2 & $2(6.06)$ & $0(0.00)$ & $0(0.00)$ & $0(0.00)$ \\
\hline Osteosarcoma subtypes, canonical & $32(96.97)$ & $35(94.59)$ & $42(97.67)$ & $46(100.00)$ \\
\hline
\end{tabular}

types, $\mathrm{N}(\%)$

Best overall response according

to RECIST 1.1

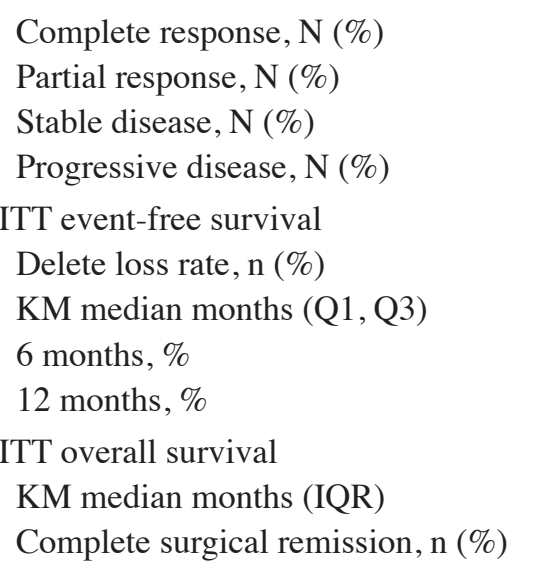

$\begin{array}{cc}0(0.0) & 0(0.0) \\ 21(63.6) & 16(43.2) \\ 12(36.4) & 8(21.6) \\ 0(0.0) & 13(35.1) \\ & \\ 9(27.3) & 11(29.7) \\ 1.4(7.5,17.1) & 4.5(3.5,6.3) \\ 78.5 \% & 36.8 \% \\ 39.5 \% & <10 \%\end{array}$

$0(0.0)$
$9(20.9)$
$26(60.5)$
$8(18.6)$

$7(16.3)$

$6.2(3.6,8.9)$

$50.9 \%$

$<10 \%$

$0(0.0)$

$13(26.1)$

$28(60.9)$

5 (10.9)

$\begin{array}{cc}19.8(9.4,51.8) & 9.9(8.0,19.0) \\ 16(48.5) & 0.0(0.0)\end{array}$
$11.3(8.1,14.8)$ $0.0(0.0)$

$12(42.9)$

$11.7(7.6,15.7)$

$71.7 \%$

$56.8 \%$

30.4 (26.9, NR) $23(50.0)$

${ }^{a}$ Other sites including lymph nodes, visceral metastasis and/or brain metastasis. ${ }^{\mathrm{b}} \mathrm{MAP} / \mathrm{I}$, including high-dose methotrexate $\left(12 \mathrm{~g} / \mathrm{m}^{2}\right)$, doxorubicin $\left(75 \mathrm{mg} / \mathrm{m}^{2}\right)$, cisplatin $\left(100-120 \mathrm{mg} / \mathrm{m}^{2}\right)$ with or without ifosfamide $\left(12 \mathrm{~g} / \mathrm{m}^{2}\right)$. AWD, alive with disease; CBR, clinical benefit rate (6 months); CI, confidence interval; DCR, disease control rate; DOD, died of disease; IE, ifosfamide and etoposide; IQR, interquartile range; ITT, intention-to-treat population; KM, Kaplan Meier; MAP/I, high-dose methotrexate, doxorubicin, cisplatin with or without ifosfamide; NED, no evidence of disease; NR, not reached; IQR, interquartile range.

major factor that influences overall survival is tumour stage. Notably, those who progressed upon more lines of systemic therapies and those with higher tumour burdens as well as more metastatic lesions and more advanced ECOG statuses had a poorer prognosis. Our study showed that apatinib plus IE chemotherapy had better disease control rate than single apatinib or apatinib in combination with camrelizumab. It is considered that these advantages are partly due to the disease control of musculoskeletal lesions by relatively high concentrations of chemotherapy drugs in the bone marrow. In the present study, a limited number of patients progressed on single apatinib and single IE chemotherapy separately could still benefit from the combination therapy, demonstrating that synergistic effects likely exist.

It is noteworthy that a considerable number of patients in the current retrospective study underwent local radiation or 
surgical resection during or after treatment. In particular, for patients treated with apatinib + IE-treated, after quick tumour regression and downstaging of the locally advanced lesions, $16 / 33(48.5 \%)$ patients received local radiation or surgery. Thus, although EFS instead of PFS was selected to evaluate disease control, whether these local treatments partly contributed to the prolonged EFS needs to be further clarified. Based on the outcomes of patients with recurrent osteosarcoma who were enrolled in seven phase II trials through the Children's Oncology Group (2), it was realized that using EFS as an end point in an osteosarcoma study was reasonable given that the historical benchmark for tumour shrinkage is difficult to obtain and detect. However, given that our past two prospective trials (11-13) used PFS as an end point without local treatments, caution should be taken when attempting to compare the outcomes of these cohorts.

Toxicity tends to be partly associated with apatinib and with IE. Given past experiences of the overall incidence of toxicity in the phase II trials of advanced osteosarcoma $(11,13,18,22,23,33-35,37)$ and our routine practice, we prefer to use lower doses of apatinib and IE instead of bolus dosing. In bolus dosing, toxicities lead to frequent interruptions that can compromise the therapeutic effect. The present study observed that a large proportion of patients could remain on the combination of the 3-day IE Q2w regimen for $>12$ months. In our opinion, the ifosfamide $1.8 \mathrm{~g} / \mathrm{m}^{2} / \mathrm{d} \mathrm{d}_{1-3}$ and etoposide $100 \mathrm{mg} / \mathrm{m}^{2} / \mathrm{d} \mathrm{d}_{1-3} \mathrm{Q} 2 \mathrm{w}$ regimen with apatinib $500 \mathrm{mg}$ po seemed to be suitable for most Chinese patients Some AEs, such as PLPS, might become more severe as a result of the combination treatment given that both apatinib and IFO can cause leukoencephalopathy.

The present study has several limitations. First, given that the present study was retrospective, selection bias was unavoidable in choosing patients who received apatinib + IE. Moreover, the comparisons of the four cohorts of patients were not controlled, and the patients had clear differences in disease status, which made the interpretation and comparison of the data difficult. Second, OS was not investigated due to the short follow-up time. Third, the histological comparative end points were different (EFS and PFS), because unlike the prospective phase 2 trials, some patients underwent lesion resection during or after treatment in the current real-world study. Moreover, given that local radiotherapy or surgeries might benefit EFS, this comparison was relatively promiscuous. We compared our synchronous retrospective data of patients who received IE chemotherapy alone with those of controls and found almost the same surgical complete remission rate $(23 / 46,50.0 \%)$, as shown in Table IV, and EFS was not significantly different retrospectively. To overcome the influence of other interventions on the outcome, we are currently performing a prospective trial to investigate this combination, from which more accurate data on this treatment strategy are expected. Fourth, the current study did not strictly perform a dose climbing ' $3+3$ ' study, which made the combination dosing prescribed according to disorder and toxicity profiles unclear.

For osteosarcoma with multiple sites of metastasis, apatinib + IE demonstrated clinically meaningful antitumour activity after failure of high-dose methotrexate, doxorubicin, cisplatin with or without ifosfamide chemotherapy or failure of former single apatinib or IE treatment, with a positive effect on delaying disease progression. The results of the present study indicated that this combination with manageable toxicity deserves further investigation in prospective trials.

\section{Acknowledgements}

The authors would like to thank Dr Danhua Shen (senior pathologist from Peking University People's Hospital) for involvement in the review of all pathological slides.

\section{Funding}

This work was supported by The Beijing Municipal Science \& Technology Project (grant no. Z181100001718054) and The Research and Development Fund of Peking University People's Hospital (Clinical Medicine $+X$ Cultivation Project, grant no. RDX2019-08).

\section{Availability of data and materials}

The datasets used and/or analysed during the current study are available from the corresponding author on reasonable request.

\section{Authors' contributions}

LX and WG conceived and designed the study. LX, JX, XS, KL, XWL, ZZ and HZ collected the data. KS reviewed the pathological slides according to RECIST 1.1. YW, JG, LX and JX clinically evaluated the efficacy of the drugs. KL, XL and XWL performed the laboratory work and statistical analysis. LX, JX and WG analysed and interpreted the data. LX wrote the manuscript. All authors confirmed the authenticity of the raw data. All authors read and approved the final manuscript.

\section{Ethics approval and consent to participate}

Both hospitals obtained approval from The Medical Ethics Committee of Peking University People's Hospital and Peking University Shougang Hospital (Beijing, China) to retrospectively review the patients' medical records and radiographic materials. Written informed consent was not required.

\section{Patient consent for publication}

Not applicable.

\section{Competing interests}

The authors declare that they have no competing interests.

\section{References}

1. Xie L, Ji T and Guo W: Anti-angiogenesis target therapy for advanced osteosarcoma (Review). Oncol Rep 38: 625-636, 2017.

2. Lagmay JP, Krailo MD, Dang H, Kim A, Hawkins DS, Beaty O III, Widemann BC, Zwerdling T, Bomgaars L, Langevin AM, et al: Outcome of patients with recurrent osteosarcoma enrolled in seven phase II trials through Children's Cancer Group, Pediatric Oncology Group, and Children's Oncology Group: Learning from the past to move forward. J Clin Oncol 34: 3031-3038, 2016. 
3. Carrle D and Bielack SS: Current strategies of chemotherapy in osteosarcoma. Int Orthop 30: 445-451, 2006.

4. Roberts RD, Lizardo MM, Reed DR, Hingorani P, Glover J, Allen-Rhoades W, Fan T, Khanna C, Sweet-Cordero EA, Cash T, et al: Provocative questions in osteosarcoma basic and translational biology: A report from the Children's Oncology Group. Cancer 125: 3514-3525, 2019.

5. Tian Z, Niu X and Yao W: Receptor tyrosine kinases in osteosarcoma treatment: Which is the key target? Front Oncol 10: 1642, 2020.

6. Shaikh AB, Li F, Li M, He B, He X, Chen G, Guo B, Li D, Jiang F, Dang L, et al: Present advances and future perspectives of molecular targeted therapy for osteosarcoma. Int J Mol Sci 17: 506, 2016.

7. Zhou W, Hao M, Du X, Chen K, Wang G and Yang J: Advances in targeted therapy for osteosarcoma. Discov Med 17: 301-307, 2014

8. Liu K, Ren T, Huang Y, Sun K, Bao X, Wang S, Zheng B and Guo W: Apatinib promotes autophagy and apoptosis through VEGFR2/STAT3/BCL-2 signaling in osteosarcoma. Cell Death Dis 8: e3015, 2017

9. Zheng B, Ren T, Huang Y and Guo W: Apatinib inhibits migration and invasion as well as PD-L1 expression in osteosarcoma by targeting STAT3. Biochem Biophys Res Commun 495: $1695-1701,2018$

10. Xie L, Guo W, Wang Y, Yan T, Ji T and Xu J: Apatinib for advanced sarcoma: Results from multiple institutions' off-label use in China. BMC Cancer 18: 396, 2018.

11. Xie L, Xu J, Sun X, Tang X, Yan T, Yang R and Guo W: Apatinib for advanced osteosarcoma after failure of standard multimodal therapy: An open label phase II clinical trial. Oncologist 24: e542-e550, 2019.

12. Correction: Apatinib plus camrelizumab (anti-PD1 therapy, SHR-1210) for advanced osteosarcoma (APFAO) progressing after chemotherapy: A single-arm, open-label, phase 2 trial. J Immunother Cancer 8: e000798corr000791, 2020.

13. Xie L, Xu J, Sun X, Guo W, Gu J, Liu K, Zheng B, Ren T, Huang Y, Tang X, et al: Apatinib plus camrelizumab (anti-PD1 therapy, SHR-1210) for advanced osteosarcoma (APFAO) progressing after chemotherapy: A single-arm, open-label, phase 2 trial. J Immunother Cancer 8: e000798, 2020.

14. Rosen G, Marcove RC, Caparros B, Nirenberg A, Kosloff C and Huvos AG: Primary osteogenic sarcoma: The rationale for preoperative chemotherapy and delayed surgery. Cancer 43 : 2163-2177, 1979.

15. Cormier JN, Patel SR, Herzog CE, Ballo MT, Burgess MA Feig BW, Hunt KK, Raney RB, Zagars GK, Benjamin RS and Pisters PW: Concurrent ifosfamide-based chemotherapy and irradiation. Analysis of treatment-related toxicity in 43 patients with sarcoma. Cancer 92: 1550-1555, 2001.

16. Miser JS, Kinsella TJ, Triche TJ, Tsokos M, Jarosinski P, Forquer R, Wesley R and Magrath I: Ifosfamide with mesna uroprotection and etoposide: An effective regimen in the treatment of recurrent sarcomas and other tumors of children and young adults. J Clin Oncol 5: 1191-1198, 1987.

17. Marina NM, Smeland S, Bielack SS, Bernstein M, Jovic G Krailo MD, Hook JM, Arndt C, van den Berg H, Brennan B, et al: Comparison of MAPIE versus MAP in patients with a poor response to preoperative chemotherapy for newly diagnosed high-grade osteosarcoma (EURAMOS-1): An open-label, international, randomised controlled trial. Lancet Oncol 17: 1396-1408, 2016

18. Goorin AM, Harris MB, Bernstein M, Ferguson W, Devidas M, Siegal GP, Gebhardt MC, Schwartz CL, Link M and Grier HE: Phase II/III trial of etoposide and high-dose ifosfamide in newly diagnosed metastatic osteosarcoma: A pediatric oncology group trial. J Clin Oncol 20: 426-433, 2002.

19. Ferrari S, Mercuri M, Picci P, Bertoni F, Brach del Prever A, Tienghi A, Mancini A, Longhi A, Rimondini S, Donati D, et al: Nonmetastatic osteosarcoma of the extremity: Results of a neoadjuvant chemotherapy protocol (IOR/OS-3) with high-dose methotrexate, intraarterial or intravenous cisplatin, doxorubicin, and salvage chemotherapy based on histologic tumor response. Tumori 85: 458-464, 1999 .

20. Salzer-Kuntschik M, Delling G, Beron G and Sigmund R: Morphological grades of regression in osteosarcoma after polychemotherapy-study COSS 80. J Cancer Res Clin Oncol 106 (Suppl): S21-S24, 1983.

21. Ferrari S, Bielack SS, Smeland S, Longhi A, Egerer G, Sundby Hall K, Donati D, Kevric M, Brosjo O Comandone A, et al: EURO-B.O.S.S.: A European study on chemotherapy in bone-sarcoma patients aged over 40: Outcome in primary high-grade osteosarcoma. Tumori 104: 30-36, 2018.
22. Gaspar N, Occean BV, Pacquement H, Bompas E, Bouvier C, Brisse HJ, Castex MP, Cheurfa N, Corradini N, Delaye J, et al Results of methotrexate-etoposide-ifosfamide based regimen (M-EI) in osteosarcoma patients included in the French OS2006/sarcome-09 study. Eur J Cancer 88: 57-66, 2018.

23. Gaspar N, Sirvent B, Venkatramani R, Longhi A, Lervat C, Casanova M, Aerts I, Bielack SS, Entz-Werle N, Strauss S, et al: 1399 Phase 1 combination dose-finding/phase 2 expansion cohorts of lenvatinib + etoposide + ifosfamide in patients (pts) aged 2 to 25 years with relapsed/refractory $(\mathrm{r} / \mathrm{r})$ osteosarcoma. Ann Oncol 30 (Suppl 5): v683-v709, 2019.

24. Enneking WF, Spanier SS and Goodman MA: A system for the surgical staging of musculoskeletal sarcoma. Clin Orthop Relat Res 153: 106-120, 1980

25. Xie L, Xu J, Dong S, Gao J, Tang X, Yan T, Yang R and Guo W: Gain and loss from transcatheter intra-arterial limb infusion of cisplatin for extremity osteosarcoma: A retrospective study of 99 cases in the past six years. Cancer Manag Res 11: 7183-7195, 2019.

26. Eisenhauer EA, Therasse P, Bogaerts J, Schwartz LH, Sargent D, Ford R, Dancey J, Arbuck S, Gwyther S, Mooney M, et al: New response evaluation criteria in solid tumours: Revised RECIST guideline (version 1.1). Eur J Cancer 45: 228-247, 2009.

27. Verger E, Salamero M and Conill C: Can Karnofsky performance status be transformed to the Eastern Cooperative Oncology Group scoring scale and vice versa? Eur J Cancer 28A: 1328-1330, 1992

28. U.S. Department of Health and Human Services: Common Terminology Criteria for Adverse Events (CTCAE) Version 5.0. https://ctep.cancer.gov/protocoldevelopment/electronic_applications/docs/ctcae_v5_quick_reference_5x7.pdf. Accessed November 27, 2018.

29. Nagy A, Lánczky A, Menyhárt O and Győrffy B: Validation of miRNA prognostic power in hepatocellular carcinoma using expression data of independent datasets. Sci Rep 8: 9227, 2018.

30. Collins M, Wilhelm M, Conyers R, Herschtal A, Whelan J, Bielack S, Kager L, Kühne T, Sydes M, Gelderblom H, et al: Benefits and adverse events in younger versus older patients receiving neoadjuvant chemotherapy for osteosarcoma: Findings from a meta-analysis. J Clin Oncol 31: 2303-2312, 2013.

31. Luetke A, Meyers PA, Lewis I and Juergens H: Osteosarcoma treatment-where do we stand? A state of the art review. Cancer Treat Rev 40: 523-532, 2014.

32. Kansara M, Teng MW, Smyth MJ and Thomas DM: Translational biology of osteosarcoma. Nat Rev Cancer 14: 722-735, 2014.

33. Italiano A, Mir O, Mathoulin-Pelissier S, Penel N, Piperno-Neumann S, Bompas E, Chevreau C, Duffaud F, Entz-Werle N, Saada E, et al: Cabozantinib in patients with advanced Ewing sarcoma or osteosarcoma (CABONE): A multicentre, single-arm, phase 2 trial. Lancet Oncol 21: 446-455, 2020.

34. Duffaud F, Mir O, Boudou-Rouquette P, Piperno-Neumann S, Penel N, Bompas E, Delcambre C, Kalbacher E, Italiano A, Collard $\mathrm{O}$, et al: Efficacy and safety of regorafenib in adult patients with metastatic osteosarcoma: A non-comparative, randomised, double-blind, placebo-controlled, phase 2 study. Lancet Oncol 20: 120-133, 2019.

35. Grignani G, Palmerini E, Dileo P, Asaftei SD, D'Ambrosio L, Pignochino Y, Mercuri M, Picci P, Fagioli F, Casali PG, et al: A phase II trial of sorafenib in relapsed and unresectable high-grade osteosarcoma after failure of standard multimodal therapy: An Italian Sarcoma Group study. Ann Oncol 23: 508-516, 2012.

36. Vasudev NS and Reynolds AR: Anti-angiogenic therapy for cancer: Current progress, unresolved questions and future directions. Angiogenesis 17: 471-494, 2014.

37. Grignani G, Palmerini E, Ferraresi V, D'Ambrosio L, Bertulli R, Asaftei SD, Tamburini A, Pignochino Y, Sangiolo D, Marchesi E, et al: Sorafenib and everolimus for patients with unresectable high-grade osteosarcoma progressing after standard treatment: A non-randomised phase 2 clinical trial. Lancet Oncol 16: 98-107, 2015.

This work is licensed under a Creative Commons Attribution-NonCommercial-NoDerivatives 4.0 International (CC BY-NC-ND 4.0) License. 